\title{
Workaround identification as an instrument for work analysis and design: a case study on ePrescription
}

\author{
Polyxeni Vassilakopoulou*, Vassilis Tsagkas and Nicolas Marmaras \\ Ergonomics Unit, School of Mechanical Engineering, National Technical University of Athens, Heroon \\ Polytechniou 9, 15780 Zografou, Greece
}

\begin{abstract}
This paper investigates workarounds occurring while pharmacists use an electronic prescription (ePrescription) application. ePrescription enables physicians to send prescriptions electronically to pharmacies and was recently introduced in Greece as a solution for controlling the ever-increasing medication costs and improving patient safety. The research was carried out in two phases: (i) review of the relevant documentation, (ii) fieldwork (field observations and semi-structured interviews with the pharmacists) in three pharmacies. The workarounds identified fall into five categories and namely: (a) bypass of ePrescription application (i.e. ePrescription application is not used at all), (b) temporal nonconformity in the use of ePrescription application (i.e. usage of the application, but not at the formally specified time), (c) catachresis of ePrescription application (i.e. usage of the application, but not according to the intended way), (d) ePrescription application substitution by another electronic application, and (e) delegation of ePrescription application usage to non-authorized users. The outcome of the research can be used for the improvement of multiple work system elements such as the tools, the procedures, the organisational arrangements and the policies.
\end{abstract}

Keywords: workarounds, ePrescription, pharmacists, work analysis

\section{Introduction}

This paper investigates workarounds occurring while pharmacists use an electronic prescription application. The outcome of this investigation can be used for the improvement of multiple work system elements such as the tools, the procedures, the organisational arrangements and the policies.

Human actors often deviate from formal procedures and follow alternative, informally devised work processes (see $[5,11])$. Such deviating behaviours usually named workarounds- are extensively studied in the Information Systems domain. Gasser [4], observed that users often "intentionally use computing in ways for which it was not designed or avoid its use and rely on an alternative means of accomplishing work". Kobayashi [8] defined workarounds as "informal temporary practices for handling exceptions to normal workflow". Similarly, Poelmans [13], Ash [1] and Vestal [16] defined workarounds as alterna- tive, from normal workflow, approaches to achieve a goal.

Several researchers attempted to further explore workarounds and search for their root causes. Day [2] identified that human actors perform workarounds due to existing constraints in the work system. Koopman and Hoffman [9] note that workarounds occur when "a path to a goal is blocked". Following Koopman and Hoffman [9], Halbesleben et. al. [5] summarizes that "for a work-around to occur, the worker must perceive some disruption or block in the system that keeps him or her from completing a task as desired". Furthermore, he categorizes the sources of blocks into: (a) policies/ laws/ regulations, (b) protocols/ guidelines, (c) work process design, (d) technology, and (e) people, in terms of restricting individual choices.

However, blocks are not the only root causes of workarounds. Poelmans [13] observed that in many cases "end-users work around the system to save time and/or efforts". Besides time or effort savings,

*Corresponding author. E-mail: xvasil@central.ntua.gr 
workarounds may be motivated by learning objectives, payoff expectations, etc. Therefore, we assume that any motivation (either intrinsic or extrinsic) may cause workarounds regardless of blocks' presence. In figure 1 we depict both blocks and motivations as root causes of workarounds.

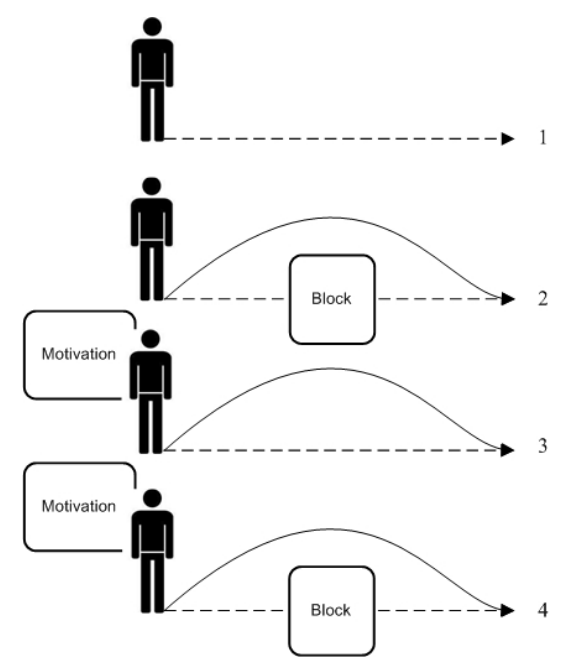

Fig. 1: 1.The dotted line represents the formal work procedure, 2.\&3.Blocks and motivations may separately cause workarounds, 4.Blocks and motivations may jointly cause workarounds

Apart from root causes, another area of interest is the exploration of workaround formulation. We assume that various factors may lead to a specific workaround formulation, including: work system's affordances, human actors' experience, physical and mental status, exploration tendencies on the work process and the impact of older work practices.

Workarounds have been analysed for different purposes and through different lenses. In his classic book on Usability Engineering, Nielsen [12] proposes the measurement of "the number of times the user had to work around an unsolvable problem" for quantifying usability and system performance. A second view on workarounds focuses on their positive effects that allow work systems to be resilient. Hovorka and Germonprez [7] view workarounds as manifestations of a "secondary design process to tailor a system to fit the user's situated tasks, metaphors, and use patterns". This view advocates system adaptability by end-users that address dynamic environments, altered plans, and non-typical work situations (e.g. [6, 14, 15]). Lauer and Rajagopalan [10] and Ferneley and Sobreperez [3] studied work- arounds as "resultant activities" of resistance to new information systems. This third view goes beyond the indication of poorly designed systems, implying that workarounds do not exist only to solve particular problems but demonstrate deeper organisational reasons that make users resist conformity to rules. We advocate that these approaches can be used as complimentary for a multilevel work analysis aiming to improve overall work effectiveness.

\section{Work system description}

Electronic prescription (or ePrescription) enables physicians to send prescriptions electronically to pharmacies. ePrescription was recently introduced in Greece as a solution for controlling the everincreasing medication costs and improving patient safety. The technical solution implemented is webbased and supports both physicians (as prescribers) pharmacists (who are responsible for processing the prescription orders). The application is accessed by its users via simple internet connections. Access is controlled at the user level (registered users go through a username and password identification process) and a central repository of all prescriptions is maintained at the national level. There are plans to grant access to citizens (so they could keep track of their own prescriptions) but this is not implemented yet. With electronic prescribing, pharmacies will no longer have to deal with illegible and incomplete hand-written orders, which are a common source of errors. The application is currently being "rolled-out" while the functionality offered is being continuously amended and improved. At this moment, the prescription system is still in hybrid mode (both electronic and manual prescribing and processing allowed) and its usage is mandatory only for prescriptions to be reimbursed by one (of the three major) health and welfare funds in Greece. Apart from the ePrescription application, most pharmacies use inventory management systems (that are "stand-alone" and do not exchange data with the newly introduced ePrescription application).

In the present paper, we focus on the process followed by the pharmacists for delivering prescribed medication to a patient. The flow chart in figure 2 gives an overview of the formal procedure pharmacists have to follow. The procedure begins right after the pharmacist receives a printed, electronically prescribed order from a patient and describes all steps required for the processing of the order. The proce- 
dure described includes all elements of the pharmacists' work system and not only the ePrescription application (e.g. it includes also the pharmacy's inventory management system).

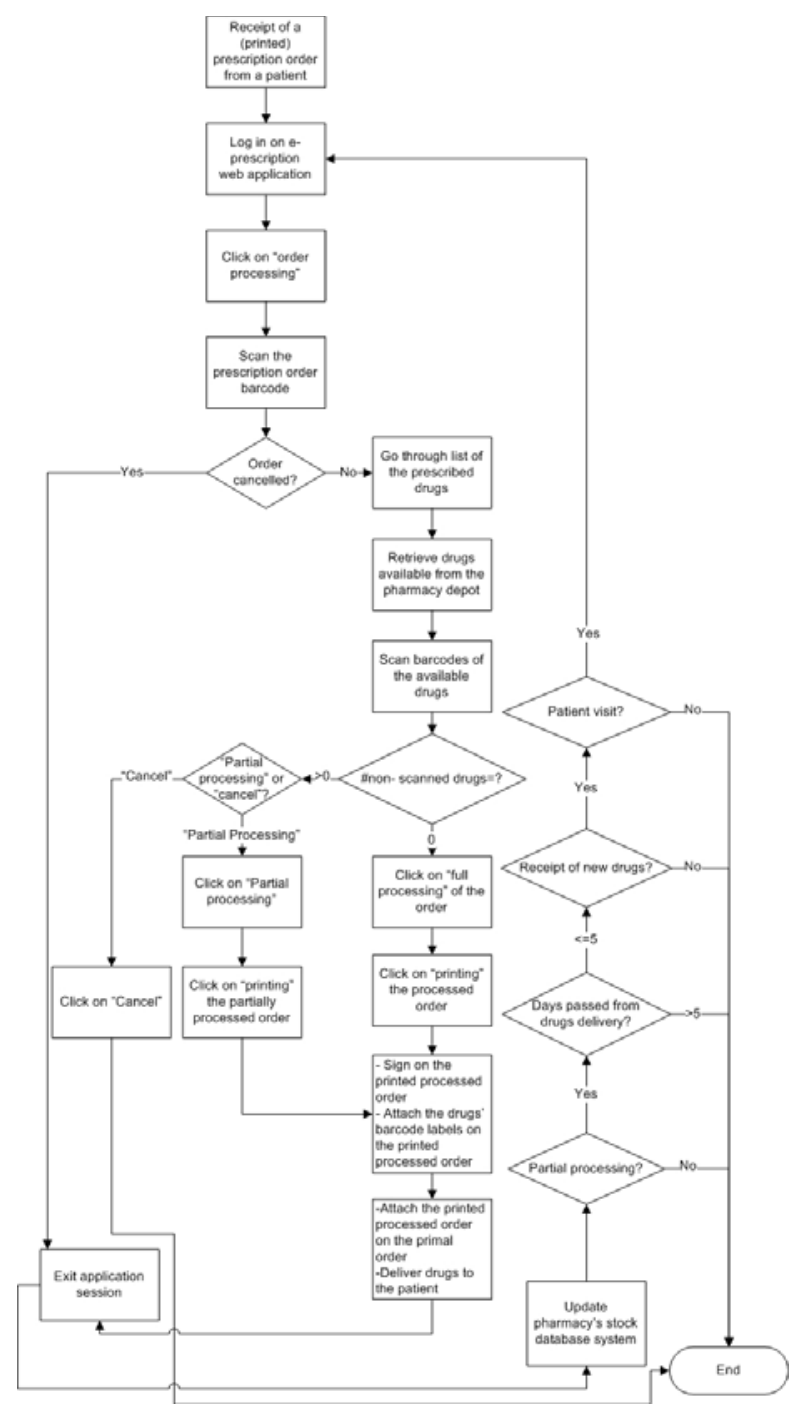

Fig. 2: The formal process pharmacists have to follow, for delivering prescribed medication to a patient

\section{Method}

Our research was carried out in two phases. At first, we reviewed the relevant documentation to the process we wanted to study. Documentation included: the electronic prescription application manuals, the Greek pharmacy legislation and guidelines, the health and welfare funds guidelines, the Health Ministry position papers and the pharmacists' code of conduct. The purpose of documentation review was twofold: to map-out the formal process that pharmacists have to follow for delivering prescribed medication to a patient and to identify the pharmacists' formal duties and obligations. The second phase of our research included fieldwork (field observations and semistructured interviews with the pharmacists) in pharmacy stores. Fieldwork was conducted in three different pharmacies over a six month period (January 2011 - June 2011).

Observations of the pharmacy personnel were directed towards the identification of workarounds on the formal process and their possible causes. The interviews were also directed towards understanding: the stances of pharmacists towards the ePrescription application, their needs and expectations, initial difficulties and they ways they overcame them. No access to personal health data was required and consequently no such data were obtained.

The pharmacists contacted were all experienced, with an average of 25 years on their profession. They were also familiar with computer applications. Two of the pharmacies were small-sized visited by an average of 50 patients per day. The third was a centrally located, mid-sized pharmacy visited by an average of 150 patients per day. Apart from the pharmacist, all three stores were staffed with 1-2 pharmacy technicians. A pharmacy technician is responsible for retrieving from the pharmacy depot the medication needed for an order to be processed and for following upon payments, always under the supervision of the pharmacist.

\section{Results}

The workarounds identified fall into five categories and namely: (a) bypass of ePrescription application (i.e. ePrescription application is not used at all), (b) temporal nonconformity in the use of ePrescription application (i.e. usage of the application, but not at the formally specified time), (c) catachresis of ePrescription application (i.e. usage of the application, but not according to the intended way), (d) ePrescription application substitution by another electronic application, and (e) delegation of ePrescription application usage to non-authorized users.

Table 1 presents a specific workaround example for each category, its root causes (blocks and/or motivations) and the factors for its formulation. In the 
paragraphs that follow the five workaround examples are described in detail.

\subsection{Bypass of ePrescription application}

According to the formal work procedure, in cases that a prescription order cannot be fully processed due to limited drug availability, the pharmacist has to register in the ePrescription application the "partial processing" of the order. When the remaining drugs become available the pharmacist is supposed to complete the order processing by entering once again the application in order to register the "full processing". Nevertheless, in such cases the pharmacists were observed to bypass this step, avoiding to register "partial processing" and when the remaining drugs become available, they complete the order, registering the "full processing". We assume that they bypass the step in order to save time and effort required for accessing the application twice. These workarounds were observed only in the two local pharmacies where there is trust and personal relationship between the pharmacist and the patient. Therefore, we assume that trust is a prerequisite for this workaround.

\subsection{Temporal nonconformity in ePrescription application use}

According to the formal work procedure the pharmacists must electronically process the order before delivering the prescribed medication. Nevertheless, pharmacists were frequently observed to deliver the prescribed medication to the patients and electronically process the order at a later time (this way, the printout produced cannot be signed by the patient as required by the formal procedure). In some cases pharmacists process all the orders received during the day after closing the pharmacy, while in other cases they process the orders in batches, whenever they have free time during the day. The frequent ePrescription system response delays and losses of internet access, results in pharmacists' uncertainty concerning the completion of their task and harms the quality of customer service. Therefore we assume that pharmacists prefer to post process the orders to avoid these impediments seeking faster patient service.

\subsection{Catachresis of ePrescription application}

According to the formal work procedure, pharmacists are allowed to use the "partial processing" option of the ePrescription application, only in three cases: (a) limited drug availability, (b) discrepancy among the recommended dosage and the prescribed quantity (c) patient refusal to take certain drugs. Pharmacists were observed, though, to resort to partial processing of an order, after identifying potential adverse drug interactions, or detecting that prescribed drugs are out of the physician's specialty. We assume that this catachresis is caused by an inconsistency between the foreseen, in the application, pharmacists' role and their own perceptions. In other words, pharmacists performing this workaround think that they have to control not only the quantity of the prescribed drugs, but also the suitability of the order.

\section{4. ePrescription substitution by another electronic application}

All pharmacists at the end of every month must submit to the health and welfare funds, lists of the patients served and the corresponding drugs delivered. During our field observations we discovered that all pharmacists printed these lists from the inventory management system and not from the ePrescription application. The ePrescription application can display a list of the patients served and the corresponding drugs delivered. Nevertheless, the list format does not match the template required. Therefore, pharmacists substitute the ePrescription application by using the inventory system, which displays the proper, according to the funds' template, lists.

\subsection{Non authorized users perform a task}

According to the formal procedure the authorized user of the ePrescription application is the pharmacist. Nevertheless, we observed that the pharmacy technicians were also processing orders. We assume that this workaround is caused by an effort to save time and provide more efficient patient services. Therefore when the pharmacy is crowded, pharmacists delegate to the available pharmacy technician the electronic order processing. 


\section{Discussion}

Our interviews and observations showed that the overall stances of pharmacists were positive to ePrescription as they benefited from improved legibility and completeness of medication orders and by system checks that prevent dispensing errors. Nevertheless, they did not use the new application exactly as foreseen by its designers: workarounds observed

Table 1

Workaround categories and specific examples with their root causes and factors behind their formulation

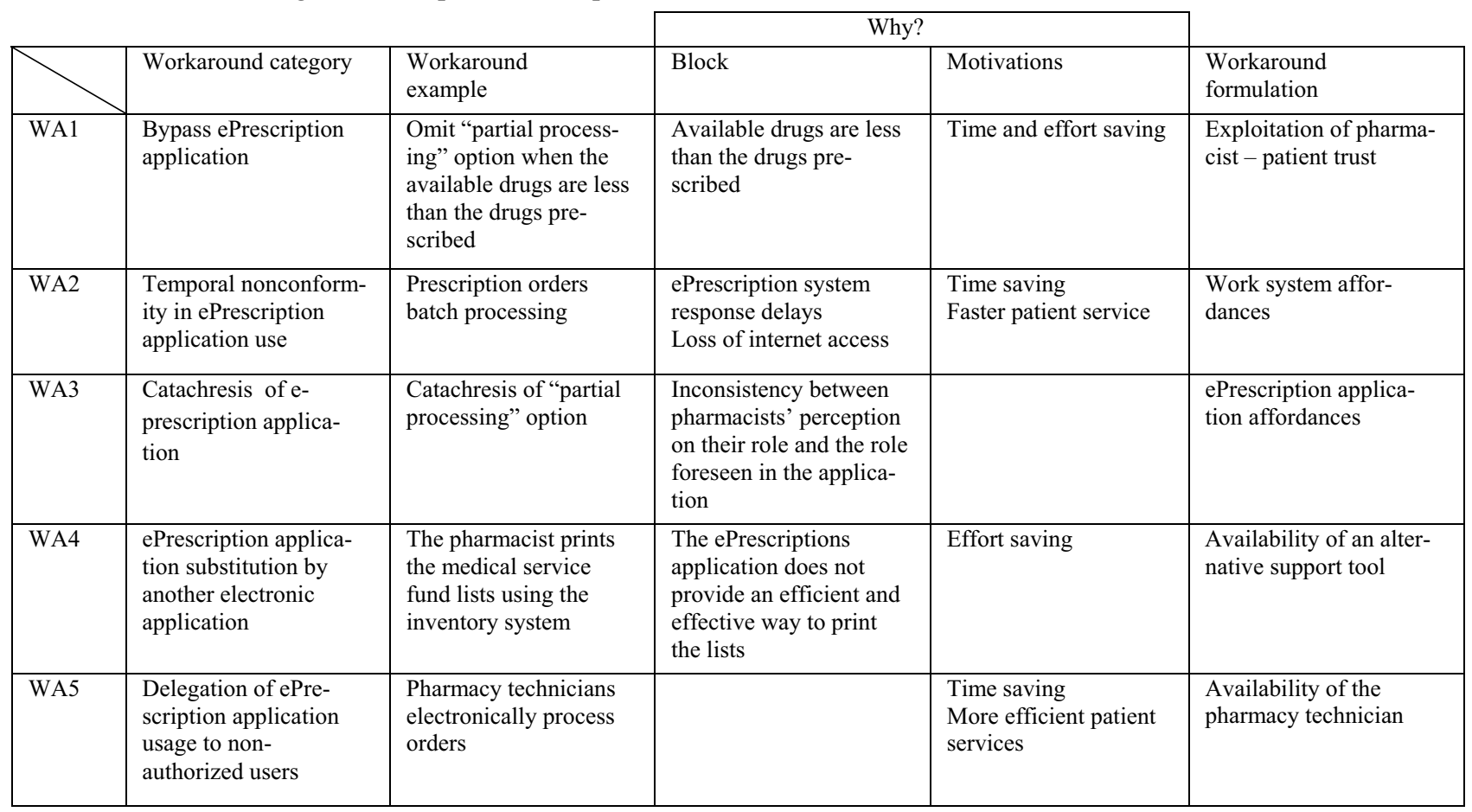

consisted of a mix of adjustments to the policy, alterations of temporal sequence, and simple substitution of the ePrescription application by alternative support tools.

Our analysis of triggering events and workaround formulation tactics revealed that perceived "blocks" in pharmacists' course of action are not always related to system's "problems" or inability to cater for all possibilities; users tend to work around whatever seems counterintuitive or counterproductive at any given moment regardless system provisions. Furthermore, even the implementation of "hard constraints" (such as the requirement of a patient's signature on the printout of the completed medication order) that are designed to establish control points and reduce process variations are not sufficient for eliminating workarounds.

Looking closer to the five examples of workarounds presented we recognize that only one of them (the workaround related to printing reports from the pharmacies inventory management application, numbered " 5 " in Table 1) can be addressed by improving the application at the interface level (designing reports that meet user needs). The other four workarounds are related either to deeper organisational reasons (numbers " 3 " and " 4 " in the table, i.e. the catachresis of the "partial completion" functionality and the delegation of authority to use the application to pharmacy technicians breaching security rules) or to the need of end users to work flexibly altering temporal requirements in the underlying logic of the application (numbers " 1 " and " 2 " in the table, i.e. the workaround related to avoiding partial processing and the one related to batch processing). Based on the above, three types of interventions can be foreseen: improvements of the interface (which can be the responsibility of the IT professionals involved), work process interventions relevant to tem- 
poral sequencing (which can be the responsibility of health and welfare funds that set the rules for prescription processing) and a reassessment of the role foreseen for pharmacists in today's environment (which would lead to clarification of their authorities and responsibilities and is something to be handled at the policy level).

Thus, our results suggest that looking at workarounds solely aiming to identify ways to improve software design results to overlooking the rich insight on the interactions among humans and other elements of the work system that they offer. This rich insight can be used for improving working conditions and overall system performance. In that sense, the analysis of workarounds is proposed as an instrument for work analysis and design that goes beyond improving software usability that can trigger innovation and ultimately, improve overall work effectiveness.

\section{Endnotes}

${ }^{1}$ It should be noted that the system only allows the pharmacy that has initiated an order processing to complete it, i.e. the patient cannot go to a different pharmacy in order to complete a partially processed order.

\section{References}

[1] J.S. Ash, M. Berg, and E. Coiera, Some unintended consequences of information technology in health care: the nature of patient care information system-related errors. Journal of the American Medical Informatics Association, 2004. 11(2): p. 104-112.

[2] D.L. Day, User responses to constraints in computerized design tools (extended abstract). SIGSOFT Softw. Eng. Notes, 1996. 21(5): p. 47-50
[3] E.H. Ferneley, and P. Sobreperez, Resist, comply or workaround? An examination of different facets of user engagement with information systems. European Journal of Information Systems, 2006. 15(4): p. 345-356.

[4] L. Gasser, The integration of computing and routine work. ACM Trans. Inf. Syst., 1986. 4(3): p. 205-225.

[5] J.R.B. Halbesleben, D.S. Wakefield, and B.J. Wakefield, Work-arounds in health care settings: Literature review and research agenda. Health Care Management Review, 2008. 33(1): p. 2-12.

[6] A. Henderson, and M. Kyng, There's no place like home: continuing design in use, in Human-computer interaction, M.B. Ronald, et al., Editors. 1995, Morgan Kaufmann Publishers Inc. p. 793-803.

[7] D.S. Hovorka, and M. Germonprez, Reflecting, Tinkering, and Tailoring: Implications for Theories of Information System Design, Computer Supported Cooperative Work, 2011, Volume 201, Part 2, p. 135-149.

[8] M. Kobayashi et al., Work coordination, workflow, and workarounds in a medical context, in CHI '05 extended abstracts on Human factors in computing systems. 2005, ACM: Portland, OR, USA. p. 1561-1564.

[9] P. Koopman and R.R. Hoffman, Work-arounds, Make-work, and Kludges. IEEE Intelligent Systems, 2003. 18(6): p. 70-75.

[10]T. Lauer, and B. Rajagopalan, Examining the relationship between acceptance and resistance in system implementation. AMCIS2002 Proceedings, 2002.

[11]C.J. McDonald, (2006). Computerization can create safety hazards: A bar-coding near miss. Annals of Internal Medi-cine, $144,510-516$.

[12]J. Nielsen Usability Engineering. 1994: Morgan Kaufmann, San Francisco.

[13]S. Poelmans, Workarounds and distributed viscosity in a workflow system: a case study. SIGGROUP Bull., 1999. 20(3): p. 11-12.

[14] M. Robinson, Design for unanticipated use, in Proceedings of the third conference on European Conference on ComputerSupported Cooperative Work. 1993, Kluwer Academic Publishers: Milan, Italy. p. 187-202.

[15] M. Robinson, and L. Bannon, Questioning representations, in Proceedings of the second conference on European Conference on Computer-Supported Cooperative Work. 1991, Kluwer Academic Publishers: Amsterdam, The Netherlands. p. 219-233.

[16] K. Vestal, Nursing and the Art of the Workaround. 2008. 6(4): p. 8-9. 